\title{
Berechnung der auftretenden lokalen Kräfte auf der magnetischen Beschichtung eines magnetischen Rasterkraftmikroskops
}

\author{
T. Preisner and W. Mathis \\ Institut für Theoretische Elektrotechnik, Leibniz Universität Hannover, Appelstr. 9A, 30167 Hannover, Germany
}

\begin{abstract}
Zusammenfassung. Die numerische Berechnung entstehender Kraftwirkungen auf Körper aufgrund magnetischer Wechselwirkungen zwischen diesen, ist in Bezug auf die mechanische Deformation dieser Körper ein noch nicht vollständig gelöstes Problem. In dieser Arbeit wird ein Vergleich vorhandener Kraftberechnungsmethoden hinsichtlich der totalen Kraft anhand eines analytisch berechenbaren Beispiels vorgestellt, sowie Unterschiede der lokalen Kraftdichten dieser Methoden in Anwendung auf die magnetische Beschichtung eines magnetischen Rasterkraftmikroskops aufgezeigt.
\end{abstract}

Abstract. Due to magnetic interactions between magnetic field inducing bodies, force effects occur on the materials. The numerical computation of those occuring forces with respect to a subsequent structural analysis of a deformable material is still a topic of interest in research. In this paper a comparison between several existent force calculation methods regarding the total force is given for an example with two magnetic cubes. Furthermore, differences are shown concerning the local force densities on the magnetic coating of a magnetic force microscope.

\section{Einleitung}

Durch die zunehmende Miniaturisierung elektronischer Komponenten in integrierten Schaltungen steigt auch ihre Fehleranfälligkeit und Zuverlässigkeit. Geeignete Messverfahren dienen bei der Synthese und Analyse dieser Nanobzw. Mikrosysteme zur Verifikation derartiger Probleme. Ein geeignetes Messinstrument ist dabei das magnetische Rasterkraftmikroskop (RKM). Der probensensitive Part dieses Mikroskops besteht aus einem mikromechanischen Federbal-

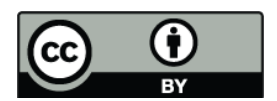

Correspondence to: T. Preisner (preisner@tet.uni-hannover.de) ken (Cantilever), an dem sich beispielsweise eine pyramidale oder konische Spitze befindet, welche in einer bestimmten Lifthöhe über eine Oberflächenstruktur rastert. Dabei wird zwischen den sogenannten Massiv- bzw. Dünnschichtsonden unterschieden. Während die Massivsonden vollständig aus ferromagnetischem Material bestehen, ist das Grundmaterial der Dünnschichtsonden typischerweise Siliziumnitrid, welches mit einem dünnen ferromagnetischen Film überzogen wird. Die Magnetisierungsrichtung der Spitze ist dabei normalerweise orthogonal zur Probenoberfläche gerichtet. Magnetische Wechselwirkungen zwischen Probenoberfläche und Spitzenmaterial führen zu einer Kraftwirkung auf den Cantilever. Durch beispielsweise optische Verfahren wird die Deformation des Cantilevers gemessen, welche direkt mit der Kraftwirkung und damit mit der magnetischen Wechselwirkung korreliert ist. Durch diese Methode können sowohl magnetische Domänen als auch elektrische Ströme, wie in $\mathrm{Pu}$ et al. (2002) gezeigt wurde, in dem Probenmaterial detektiert werden. Mess- bzw. Interpretationsschwierigkeiten treten allerdings beispielsweise bei ebenen Magnetisierungen der Probe auf, da dabei die Magnetisierungen der Spitze und Probe orthogonal zueinander sind und es somit zu keiner Kraftwirkung kommt. Des Weiteren kann insbesondere bei weichferromagnetischen Probenmaterialen die Beeinflussung des Cantileverspitzenfeldes auf die Probe deart destruktiv sein, dass einzelne magnetische Domänen in der Probe hinter der Scanrichtung der Spitze hergezogen werden. Dies kann bis zur Zerstörung der magnetischen Probeninformation führen. Weiterhin entstehen Messungenauigkeiten durch geometrische Asymmetrien und Materialheterogenitäten der Spitze. Diese Umstände können durch theoretische Betrachtungen im Vorfeld vermieden bzw. im Anschluss an eine Messung herausgerechnet werden. Für die Simulation des magnetischen Rasterkraftmikroskops kann das Modell in zwei Teile gegliedert werden, welche das magnetische bzw. das mechanische Verhalten beschreiben. In der vorgestellten Arbeit wird aufbauend zu der Finiten Elemente 


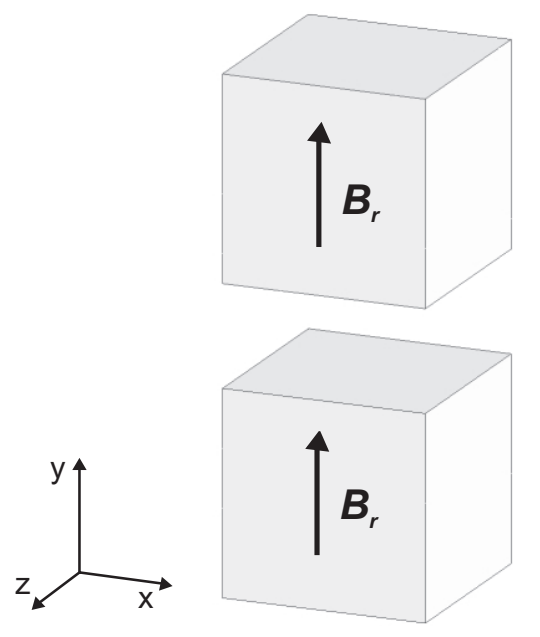

Abb. 1. Anordnung zweier permanentmagnetischer Blöcke.

Methode der Teilaspekt der Kraftberechnung auf die magnetische Cantileverspitze eines magnetischen Rasterkraftmikroskops näher beleuchtet. Dieser Aspekt ist insbesondere wichtig, um die Kopplung zwischen dem magnetischen und mechanischen Modell zu gewährleisten. Dazu wurden fünf verschiedene Kraftberechnungsmethoden implementiert und bezüglich der totalen Kraft sowie den lokalen Kraftdichten, welche auf die magnetische Spitze des Cantilevers wirken, miteinander verglichen.

\section{Kraftberechnungsmethoden}

Im Folgenden werden die zur Kraftberechnung implementierten Verfahren kurz vorgestellt.

\subsection{Methode äquivalenter magnetischer Quellen}

Eine Methode zur Kraftberechnung permanentmagnetischer Materialien besteht darin, diese durch äquivalente magnetische Quellen zu ersetzen. Wie beispielsweise in Jackson (1975) gezeigt, können diese Materialien durch eine Anordnung einer Volumenstromdichte $\boldsymbol{J}_{v}=\nabla \times \boldsymbol{M}$ und einer Oberflächenstromdichte $\boldsymbol{J}_{s}=-\boldsymbol{n} \times \boldsymbol{M}$ ersetzt werden, wobei $\boldsymbol{M}$ die Magnetisierung des Materials und $\boldsymbol{n}$ der nach außen gerichtete Einheitsnormalenvektor der Oberfläche ist. Ist die Magnetisierung über dem Körper homogen, hat die Volumenstromdichte keinen Beitrag zur Gesamtstromdichte. Die Oberflächenkraftdichte kann somit über die Lorentzformel

$\boldsymbol{f}_{s}=\boldsymbol{J}_{s} \times \boldsymbol{B}_{s}$

berechnet werden.

Wie in Jackson (1975) dargestellt, besteht auch die Möglichkeit fiktive magnetische Ladungen als äquivalente Quellen zu nutzen. Dabei wird die Magnetisierung durch

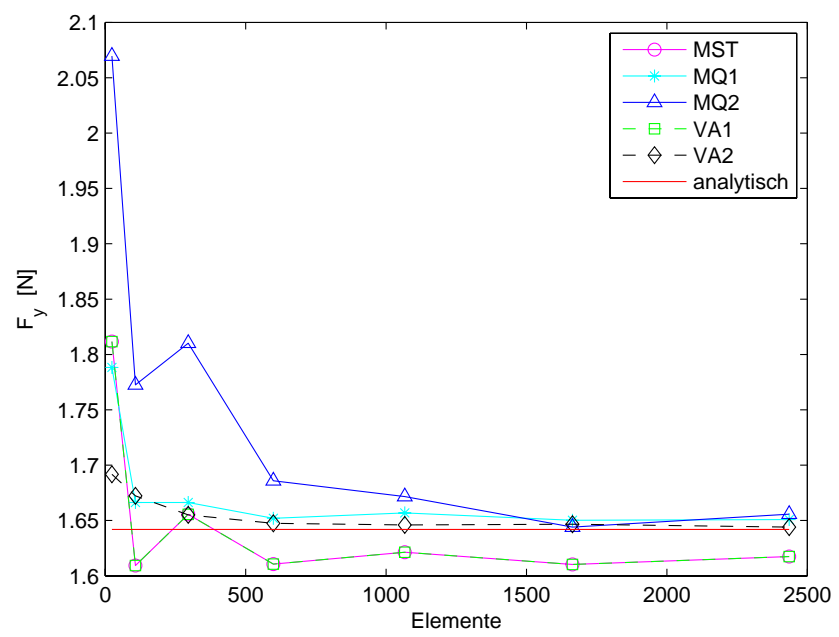

Abb. 2. Verlauf der Anziehungskraft $F_{y}$ auf den unteren magnetischen Würfel verschiedener Kraftberechnungsmethoden in Abhängigkeit der Diskretisierung eines Würfels.

eine geeignete Volumenladungsdichte $\rho_{v}=-\mu_{0}(\nabla \cdot \boldsymbol{M})$ und Oberflächenladungsdichte $\rho_{s}=\mu_{0}(\boldsymbol{n} \cdot \boldsymbol{M})$ ersetzt. Für den Fall einer homogenen Magnetisierung verschwindet die Volumenladungsdichte und die Oberflächenkraftdichte ergibt sich durch

$\boldsymbol{f}_{s}=\rho_{s} \cdot \boldsymbol{H}_{s}$.

Mit einer Diskretisierung des Problemgebietes $\Omega$ mit finiten Elementen lässt sich die lokale Kraftdichte an der Oberfläche eines Elementes $e$ berechnen, indem das arithmetische Mittel aus den zwei an der Oberfläche angrenzenden Elementen $e_{-}$ und $e_{+} \boldsymbol{B}$ - bzw. $\boldsymbol{H}$-Feldkomponenten analog zu De Medeiros et al. (1999) genommen wird. Die Gleichungen (1) und (2) lassen sich dann wie folgt ausdrücken:

$$
\begin{gathered}
\boldsymbol{f}_{s}=\boldsymbol{J}_{s} \times\left(\frac{\boldsymbol{B}_{s-}+\boldsymbol{B}_{s+}}{2}\right) \\
\boldsymbol{f}_{s}=\rho_{s} \cdot\left(\frac{\boldsymbol{H}_{s-}+\boldsymbol{H}_{s+}}{2}\right) .
\end{gathered}
$$

\subsection{Maxwellscher Spannungstensor}

Ein klassischer Ansatz, um die Kraft auf ein ferromagnetisches Material mit dem Maxwellschen Spannungstensor (MST) zu berechnen, ist dieses Gebiet so durch geeignete Stromdichten zu ersetzen, dass sich das äußere Feld nicht ändert (Salon et al., 1995). Mit Hilfe der Lorentz Volumenkraftdichte $\boldsymbol{p}_{v}=\boldsymbol{J} \times \boldsymbol{B}$ und dem Ampere'schen Gesetz für magnetostatische Feldzusammenhänge $\nabla \times \boldsymbol{H}=\boldsymbol{J}$ lässt sich $\boldsymbol{p}_{v}$ als Divergenz eines Tensors $\boldsymbol{T}^{* 1}$ umformulieren. Dieser

\footnotetext{
${ }^{1}$ Das Asterisk-Symbol (*) kennzeichnet hier einen Tensor.
} 
Tabelle 1. Zahlenwerte der Anziehungskraft $F_{y}$ auf den unteren Würfel im Vergleich zur analytischen Lösung.

\begin{tabular}{c|c|cccccccccc}
\hline Elemente & $\begin{array}{c}\text { Analytisch } \\
{[\mathrm{N}]}\end{array}$ & $\begin{array}{c}\text { MST } \\
{[\mathrm{N}]}\end{array}$ & $\begin{array}{c}\text { Fehler } \\
{[\%]}\end{array}$ & $\begin{array}{c}\text { MQ1 } \\
{[\mathrm{N}]}\end{array}$ & $\begin{array}{c}\text { Fehler } \\
{[\%]}\end{array}$ & $\begin{array}{c}\text { MQ2 } \\
{[\mathrm{N}]}\end{array}$ & $\begin{array}{c}\text { Fehler } \\
{[\%]}\end{array}$ & $\begin{array}{c}\text { VA1 } \\
{[\mathrm{N}]}\end{array}$ & $\begin{array}{c}\text { Fehler } \\
{[\%]}\end{array}$ & $\begin{array}{c}\text { VA2 } \\
{[\%]}\end{array}$ & $\begin{array}{c}\text { Fehler } \\
{[\%]}\end{array}$ \\
\hline 24 & 1.642 & 1,812 & 9,36 & 1,788 & 8,18 & 2,070 & 20,7 & 1,812 & 9,36 & 1,692 & 2,94 \\
599 & 1.642 & 1,611 & $-1,95$ & 1,652 & 0,60 & 1,686 & 2,60 & 1,611 & $-1,95$ & 1,647 & 0,33 \\
2436 & 1.642 & 1,618 & $-1,52$ & 1,651 & 0,54 & 1,656 & 0,83 & 1,618 & $-1,52$ & 1,644 & 0,13 \\
\hline
\end{tabular}

Tensor hat nach der beschriebenen Vorgehensweise für den magnetostatischen Fall die Form

$$
\boldsymbol{T}^{*}=\frac{1}{\mu_{0}}\left[\begin{array}{ccc}
B_{x}^{2}-\frac{1}{2}|\boldsymbol{B}|^{2} & B_{x} B_{y} & B_{x} B_{z} \\
B_{y} B_{x} & B_{y}^{2}-\frac{1}{2}|\boldsymbol{B}|^{2} & B_{y} B_{z} \\
B_{z} B_{x} & B_{z} B_{y} & B_{z}^{2}-\frac{1}{2}|\boldsymbol{B}|^{2}
\end{array}\right] .
$$

Die totale Kraft auf das ferromagnetische Objekt wird nun durch Integration der Divergenz des Tensor $\boldsymbol{T}^{*}$ über das Gebiet $\Omega$ berechnet. Durch die Anwendung des Integralsatzes von Gauss kann das Volumenintegral in ein Flächenintegral über die geschlossene Hülle transformiert werden.

$$
\boldsymbol{F}=\int_{\Omega} \operatorname{div} \boldsymbol{T}^{*} d \Omega=\oint_{\Gamma} \boldsymbol{T}^{*} d \boldsymbol{\Gamma}
$$

Wie in den Arbeiten von Benhama et al. (1996) gezeigt wurde, hängt die Güte der Kraftlösung über den MST stark von der verwendeten Integrationshülle ab. Hier wurde ein Integrationsweg gewählt, welcher den Mittelpunkt der Elemente und die jeweilige Flächen- bzw. Kantenmitte dieser schneidet.

\subsection{Das Prinzip der virtuellen Arbeit}

Das Prinzip der virtuellen Arbeit oder der virtuellen Verschiebung geht für einen Finite Elemente Ansatz elektromagnetischer Feldprobleme auf Coulomb (1983) zurück. Eine virtuelle Verschiebung des betrachteten Körpers hat eine Energieänderung in diesem Gebiet zur Folge, welche mit der auf den Körper wirkenden Kraft in dieser Richtung korreliert ist. Die Energie eines permanentmagnetischen Materials kann dabei durch

$W=\frac{1}{2 \mu_{0}} \int_{\Omega}\left(\boldsymbol{B}-\boldsymbol{B}_{r}\right)\left(\boldsymbol{B}-\boldsymbol{B}_{r}\right) d \Omega$

beschrieben werden (Ida und Bastos, 1992), wobei $\boldsymbol{B}_{r}$ die Remanenzinduktion ist. In einem Finite Elemente Ansatz ist das Gebiet $\Omega$ in einzelne Teilgebiete $e$ gegliedert. Eine lokale Verschiebung eines Knoten $k$ in eine Richtung $i$ führt zu einer Variation der Energie $W$ in den umliegenden Teilgebieten. Die lokale Knotenkraft $F_{i k}$ ergibt sich dann durch die Summe über die variierte Energie der umliegenden Elemente

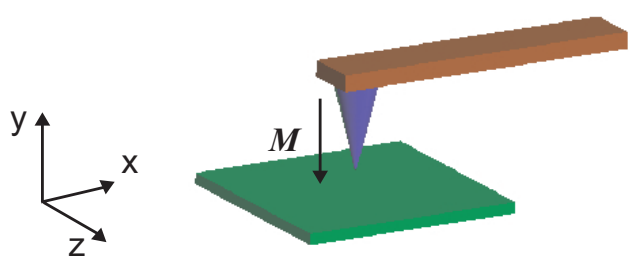

Abb. 3. Das Modell des Cantilevers mit Spitze eines magnetischen Rasterkraftmikroskops.

(De Medeiros et al., 1998)

$$
\begin{aligned}
F_{i k} & =-\sum_{e_{k}}\left[\int_{\Omega_{e_{k}}} \frac{\left(\boldsymbol{B}-\boldsymbol{B}_{r}\right)}{\mu_{0}} \mathcal{J}^{-1} \frac{\delta \mathcal{J}}{\delta s_{i}} \boldsymbol{B}|\mathcal{J}| d \Omega_{e_{k}}\right. \\
& \left.+\int_{\Omega_{e_{k}}} \frac{\left(\boldsymbol{B}-\boldsymbol{B}_{r}\right)\left(\boldsymbol{B}-\boldsymbol{B}_{r}\right)}{2 \mu_{0}} \frac{\delta|\mathcal{J}|}{\delta s_{i}} d \Omega_{e_{k}}\right] .
\end{aligned}
$$

In Gleichung (8) ist $\mathcal{J}$ die Jacobimatrix und $|\mathcal{J}|$ die Jacobideterminante.

Ein weiterer auf dem Prinzip der virtuellen Arbeit basierender Ansatz wurde erstmals von De Medeiros et al. (1998) vorgestellt. Bei diesem Ansatz wird zur Kraftberechnung nur die wechselwirkende Energie $W_{w w}$ zwischen den felderzeugenden Körpern betrachtet, d.h. der Beitrag welcher von der intrinsischen Energie $W_{\text {intr. }}$ herrührt wird von der Gesamtenergie $W$ abgezogen. Dieser Ansatz führt auf die folgende lokale Kraft am Knoten $k$ gerichtet in $i$

$$
\begin{aligned}
F_{w w, i k} & =F_{i k}-F_{\text {intr.,ik }} \\
& =-\sum_{e_{k}}\left[\int_{\Omega_{e_{k}}} \frac{\left(\boldsymbol{B}-\boldsymbol{B}_{r}\right)}{\mu_{0}} \mathcal{J}^{-1} \frac{\delta \mathcal{J}}{\delta s_{i}} \boldsymbol{B}|\mathcal{J}| d \Omega_{e_{k}}\right. \\
& -\int_{\Omega_{e_{k}}} \frac{\left(\boldsymbol{B}_{L}-\boldsymbol{B}_{r}\right)}{\mu_{0}} \mathcal{J}^{-1} \frac{\delta \mathcal{J}}{\delta s_{i}} \boldsymbol{B}_{L}|\mathcal{J}| d \Omega_{e_{k}} \\
& -\int_{\Omega_{e_{k}}} \frac{\boldsymbol{B}\left(2 \boldsymbol{B}_{r}-\boldsymbol{B}\right)}{2 \mu_{0}} \frac{\delta|\mathcal{J}|}{\delta s_{i}} d \Omega_{e_{k}} \\
& \left.+\int_{\Omega_{e_{k}}} \frac{\boldsymbol{B}_{L}\left(2 \boldsymbol{B}_{r}-\boldsymbol{B}_{L}\right)}{2 \mu_{0}} \frac{\delta|\mathcal{J}|}{\delta s_{i}} d \Omega_{e_{k}}\right]
\end{aligned}
$$

wobei $\boldsymbol{B}_{L}$ die magnetische Induktion des permanentmagnetischen Materials allein betrachtet in Luft ist. 

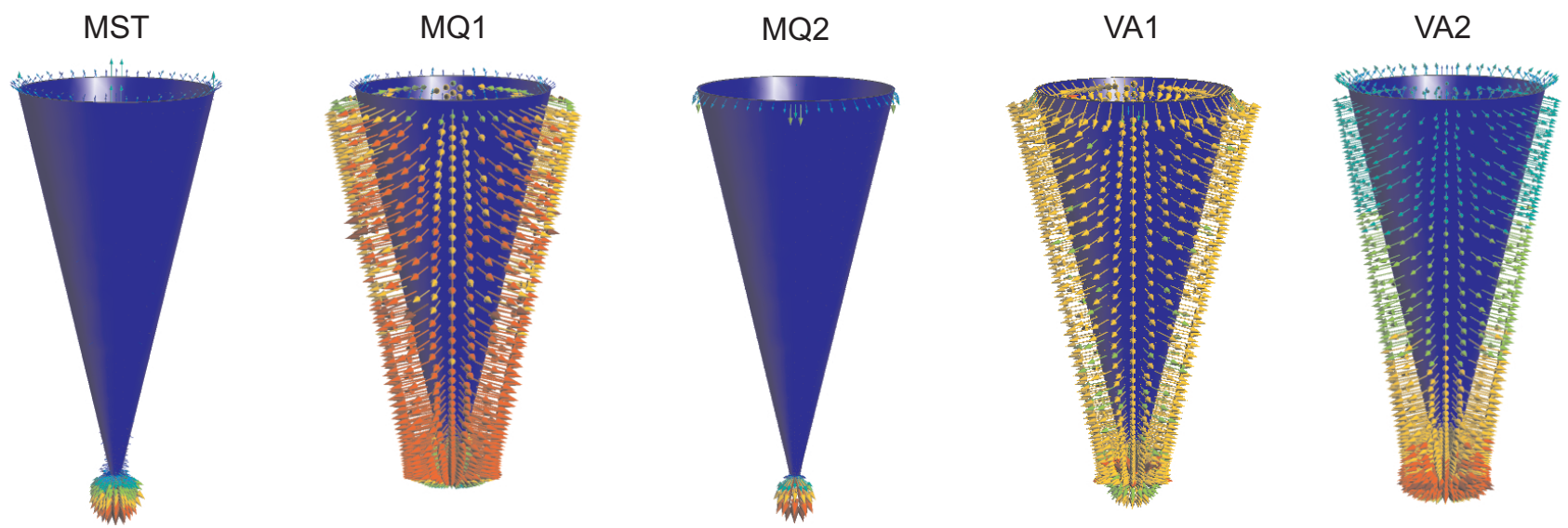

Abb. 4. Normalkomponenten der lokalen Kraftdichten auf der magnetischen Beschichtung des Rasterkraftmikroskops.

\section{Ein numerisches Beispiel}

Um qualitative Aussagen über die Effizienz der vorgestellten Methoden treffen zu können, werden diese anhand eines Beispiels mit zwei permanentmagnetischen Würfeln verifiziert (Abb. 1). Beide Körper haben ein Volumen von $1 \mathrm{~cm}^{3}$ und eine Remanenzinduktion von $\boldsymbol{B}_{r}=\left[\begin{array}{lll}0 & 0,5 & 0\end{array}\right]^{T}$ T. Der Abstand zwischen den Körpern beträgt $5 \mathrm{~mm}$. Für dieses Problem existiert nach Akoun und Yonnet (1984) eine analytische Lösung. Die numerische Kraftberechnung wurde durch eine Einteilung dieser Anordnung in finite Elemente durchgeführt und der analytischen Lösung für verschieden feine Stufen der Elementdiskretisierung eines Würfels gegenübergestellt (Tabelle 1). Bei einer sehr groben Diskretisierung von 24 Elementen pro Würfel ist das Ergebnis über die Methode äquivalenter magnetischer Ladungen (MQ2) mit einer Abweichung von 20,7\% stark fehlerhaft. Das Ergebnis über die äquivalenten magnetischen Stromdichten (MQ1) ist für die niedrige Elementanzahl mit einer Abweichung von 8,18\% deutlich besser. Die Kraftberechnung über die virtuelle $\mathrm{Ar}$ beit (VA1) und den Maxwellschen Spannungstensor (MST) ist nahezu identisch und weicht vom analytisch exakten Wert um jeweils 9,36\% ab. Das beste Ergebnis liefert die Methode der virtuellen Arbeit unter Berücksichtigung der intrinsischen Energie (VA2). Hier beträgt die Abweichung 2,94\%. Mit zunehmenden Elementanzahl pro magnetischem Würfel zeigt sich, dass die numerisch berechnete Kraft aller Methoden gegen den analytischen Wert konvergieren (Abb. 2). Die beste Annäherung bei einer Diskretisierung von 2436 Elementen mit einem Fehler von 0,13\% liefert auch hier die Methode VA2.

\section{Die lokalen Kraftdichten auf der magnetischen Be- schichtung von einem RKM}

Um ein komplettes theoretisches Modell eines magnetischen Rasterkraftmikroskops zu erstellen, muss das elektromagnetische Teilmodell mit dem mechanischen gekoppelt werden. Für die mechanische FEM-Analyse sind die mit dem elektromagnetischen Modell berechneten lokalen Kräfte als vorgegebene Randbedingungen essentiell. Wie im vorangegangenen Abschnitt gezeigt wurde, sind alle Kraftberechnungsmethoden bei geeigneter Diskretisierung für die Kraftberechnung eines permamentmagnetischen Materials anwendbar. Anders als für die totale Kraft weichen allerdings die lokalen Kräfte dieser fünf Methoden stark voneinander ab, was im Folgenden anhand der magnetischen Beschichtung von einem magnetischen RKM gezeigt wird.

\subsection{Das elektromagnetische Modell von einem RKM}

Ein einfaches Modell des magnetischen Rasterkraftmikroskops ist in Abb. 3 zu sehen. Als Proben- und Beschichtungsmaterial wurde hier eine CoCr-Legierung mit einer Magnetisierung von $\boldsymbol{M}=\left[\begin{array}{lll}0 & -749 & 0\end{array}\right]^{T} \frac{\mathrm{kA}}{\mathrm{m}}$ gewählt. Die Spitze hat einen oberen Radius von $2 \mu \mathrm{m}$ und eine Höhe von $8 \mu \mathrm{m}$. Das Verhältnis der Dicke der magnetischen Beschichtung und des Radius beträgt $\frac{1}{40}$. Des Weiteren ist der Abstand zwischen Spitze und Probenoberfläche $1,5 \mu \mathrm{m}$.

\subsection{Ergebnisse der lokalen Kräfte}

Die Ergebnisse der lokalen Kraftberechnung zeigen eine starke Abweichung im Feldbild der einzelnen Methoden. Diesen Zusammenhang zeigt Abb. 4, in welcher die Normalenkomponente der Kraftdichte in Bezug auf die Beschichtungsoberfläche der Spitze aller beschriebenen Kraftberechnungsmethoden gezeigt ist. Während sich bei der Berechnung über den MST und MQ2 ein starkes Maximum der 
lokalen Kraftdichten an der Spitze der RKM-Beschichtung ausprägt, ist bei den Methoden MQ1 und VA1 eher eine homogene Kraftdichteverteilung zu erkennen. Die virtuelle Arbeit unter Berücksichtigung der intrinsischen Energie (VA2) hat ebenfalls ein Kraftdichtemaximum an der Spitze der Beschichtung. Allerdings ist es hierbei nicht so dominant ausgeprägt wie bei den Ergebnissen über die Kraftberechnungsmethoden MST und MQ2. Für eine anschließende mechanische Deformationsanalyse des Cantilevermaterials stehen somit fünf unterschiedliche Kraftfelder zur Verfügung. Einen Ansatz, welche Kraftdichteverteilung für eine nachfolgende mechanische Analyse anwendbar ist, ist bei Barre et al. (2006) zu finden, welche ein magnetomechanisches Problem berechnet und mit experimentellen Daten verglichen haben. Die beste numerische Annäherung zu den Messdaten wurde mit der Methode der virtuellen Arbeit (VA1) erzielt. In Arbeiten von Lee et al. (2000) wird wiederum vermutet, dass die Methode der äquivalenten magnetischen Ladungen (MQ2) eine geeignete wäre, um die mechanische Analyse durchzuführen. In dem vorliegenden Problem des magnetischen Rasterkraftmikroskops tritt eine magnetische Wechselwirkung fast ausschließlich mit den letzten Atomen an der Beschichtung auf. Daher ist an dieser Stelle das magnetische Feldmaximum zu finden, und somit auch die größte Kraftdichte zu erwarten. Demnach ist die Vermutung aufzustellen, dass für diese Problemstellung die Verfahren MST, MQ2 sowie VA2 geeignet wären. Dies ist in einer folgenden Arbeit zu klären.

\section{Diskussion}

Das durch Akoun und Yonnet (1984) analytisch berechenbare Beispiel mit zwei permanentmagnetischen Würfeln hat gezeigt, dass die Kraftberechnung über die äquivalenten magnetischen Ladungen stark von der Diskretisierung des Problems abhängt. Mit einer groben Einteilung des Magneten in finite Elemente ist diese Kraftlösung mit einer $\mathrm{Ab}$ weichung von $20,7 \%$ stark fehlerbelastet. Die Berechnungen über die äquivalenten magnetischen Stromdichten, über den Maxwellschen Spannungstensor und über die beiden Verfahren der virtuellen Arbeit liefern dagegen mit Abweichungen zwischen 3\% und 10\% relativ gute Annäherungen. Mit zunehmender Elementanzahl konvergieren alle Methoden gegen den analytischen Kraftwert, wobei der Maxwellsche Spannungstensor und das Prinzip der virtuellen Arbeit mit einer feineren Diskretisierung eine recht langsame Konvergenz zeigen. Als effizienteste Methode hat sich das Verfahren der virtuellen Arbeit unter Berücksichtigung der intrinsischen Energie herausgestellt.

Bei der Untersuchung der lokalen Kraftdichten anhand des Beispiels des magnetischen Rasterkraftmikroskops zeigten sich auf der magnetischen Beschichtung der Spitze des Cantilevers fünf voneinander abweichende Feldverteilungen. Wie beschrieben wurde, kann allerdings durch Betrach- tung realer Eigenschaften zwischen Spitze und Probenmaterial hier die These aufgestellt werden, dass für eine anschließende mechanische Deformationsanalyse des Cantilever die Kraftlösungen über den Maxwellschen Spannungstensor, über die äquivalenten magnetischen Ladungen sowie über die virtuelle Arbeit unter Berücksichtigung der intrinsischen Energie vermutlich die genausten Ergebnisse der Cantileververformung liefern werden. Dies muß in einer folgenden Arbeit näher untersucht werden.

\section{Literatur}

Akoun, G. and Yonnet, J. P.: 3D analytical calculation of the forces exerted between two cuboidal magnets, IEEE T. Magn., 20, 1962-1964, 1984.

Barre, O., Brochet, P., and Hecquet, M.: Experimental validation of magnetic and electric local force formulations associated to energy principle, IEEE T. Magn., 42, 1475-1478, 2006.

Benhama, A., Williamson, A. C., and Reece, A. B. J.: Computation of electromagnetic forces from finite element field solutions, in: Proceedings of the 3rd International Conference on Computation Electromagnetics, Bath, UK, 247-252, 1996.

Coulomb, J. L.: A methodology for the determination of global electromechanical quantities from a finite element analysis and its application to evaluation of magnetic forces, torques and stiffness, IEEE T. Magn., 19, 2514-2519, 1983.

De Medeiros, L. H., Reyne, G., Meunier, G., and Yonnet, J. P.: Distribution of electromagnetic force in permanent magnets, IEEE T. Magn., 34, 3012-3015, 1998.

De Medeiros, L. H., Reyne, G., and Meunier, G.: About the distribution of forces in permanent magnets, IEEE T. Magn., 35, 1215-1218, 1999.

Ida, N. and Bastos, J. P. A.: Electromagnetics and Calculation of Fields, Springer-Verlag, New York, 1992.

Jackson, J. D.: Classical Electrodynamics, Second Edition, John Wiley \& Son, Inc., New York, 1975.

Kabashima, T., Kawahara, A., and Goto, T.: Force calculation using magnetizing currents, IEEE T. Magn., 24, 451-454, 1988.

Lee, S.-H., Park, I.-H., and Lee, K.-S.: Comparison of mechanical deformations due to different force distributions of two equivalent magnetization models, IEEE T. Magn., 34, 1368-1372, 2000.

Pu, A., Rahman, A., Thomson, D. J., and Bridges, G. E.: Magnetic force microscopy measurement of current on integrated circuits, in: Proceedings of the 2002 IEEE Canadian Conference on Electrical and Computer Engineering, CCECE 2002, 439-444, 2002.

Salon, S. J., Slavik, C. J., DeBortoli, M. J., and Reyne, G.: Analysis of Magnetic Vibrations in Rotating Electric Machines, in: Finite Elements, Electromagnetics and Design, edited by: Ratnajeevan, S. and Hoole, H., Elsevier, Amsterdam, 1995. 